\title{
Beam halo collimation in heavy ion synchrotrons
}

\author{
I. Strašík, ${ }^{1}$ I. Prokhorov, ${ }^{1,2}$ and O. Boine-Frankenheim ${ }^{1,2}$ \\ ${ }^{1}$ GSI Helmholtzzentrum für Schwerionenforschung, Planckstraße 1, 64291 Darmstadt, Germany \\ ${ }^{2}$ Technische Universität Darmstadt, Schloßgartenstraße 8, 64289 Darmstadt, Germany
}

(Received 27 June 2014; published 5 August 2015)

\begin{abstract}
This paper presents a systematic study of the halo collimation of ion beams from proton up to uranium in synchrotrons. The projected Facility for Antiproton and Ion Research synchrotron SIS100 is used as a reference case. The concepts are separated into fully stripped (e.g., ${ }^{238} \mathrm{U}^{92+}$ ) and partially stripped (e.g., ${ }^{238} \mathrm{U}^{28+}$ ) ion collimation. An application of the two-stage betatron collimation system, well established for proton accelerators, is intended also for fully stripped ions. The two-stage system consists of a primary collimator (a scattering foil) and secondary collimators (bulky absorbers). Interaction of the particles with the primary collimator (scattering, momentum losses, and nuclear interactions) was simulated by using FLUKA. Particle-tracking simulations were performed by using MAD-X. Finally, the dependence of the collimation efficiency on the primary ion species was determined. The influence of the collimation system adjustment, lattice imperfections, and beam parameters was estimated. The concept for the collimation of partially stripped ions employs a thin stripping foil in order to change their charge state. These ions are subsequently deflected towards a dump location using a beam optical element. The charge state distribution after the stripping foil was obtained from GLOBAL. The ions were tracked by using MAD-X.
\end{abstract}

DOI: 10.1103/PhysRevSTAB.18.081001

PACS numbers: 29.20.dk, 41.75.-i, 41.85.Ew, 41.85.Si

\section{INTRODUCTION}

Various beam physics mechanisms can cause particles to enter into unstable orbits with large betatron amplitudes, which leads to the formation of a beam halo and emittance growth [1-3]. The main sources of the beam halo are space charge, mismatched beam, nonlinear forces due to magnet errors, rf noise, intrabeam scattering, resonances, beambeam effects, and electron clouds [1-3]. Beam halo is one of the reasons for uncontrolled beam losses interacting with the accelerator structure [1-4]. Uncontrolled beam losses cause various problems such as residual activation of the accelerator components, quench of the superconducting magnets, vacuum degradation due to beam-induced desorption, radiation damage, e.g., of insulation materials, and background in experiments [1-8]. The main purpose of the collimation system is to remove the halo and, consequently, to reduce the aforementioned problems and to provide a well-defined and shielded dump location for the beam losses $[1,4]$.

The halo collimation system in the projected SIS100 synchrotron of Facility for Antiproton and Ion Research (FAIR) must be capable to collimate various ion species from protons up to uranium $[9,10]$. The task is even more challenging due to operation with fully (e.g., ${ }^{238} \mathrm{U}^{92+}$ ) and partially (e.g., ${ }^{238} \mathrm{U}^{28+}$ ) stripped ions. In the case of the

Published by the American Physical Society under the terms of the Creative Commons Attribution 3.0 License. Further distribution of this work must maintain attribution to the author(s) and the published article's title, journal citation, and DOI. proton and light ion beam operation, the collimation system should mainly reduce the uncontrolled residual activation and avoid quenches [5,6]. A tolerable level of uncontrolled beam losses is $1 \mathrm{~W} / \mathrm{m}$ for protons, and it is increasing with increasing ion mass [5]. For heavy ion beam operation, the collimation system is needed, in particular, to prevent the vacuum degradation and the radiation damage $[7,8]$.

For protons and fully stripped ions, a conventional twostage (or multistage) betatron collimation system $[11,12]$ is going to be applied in SIS100. Such a concept is well established and widely used for collimation of proton beams in circular accelerator facilities such as CERN LHC [13-15], J-PARC synchrotrons [16,17], Fermilab synchrotrons [18,19], ORNL SNS [20], DESY HERA $[21,22]$, or CSNS RCS [23,24]. However, there are only a few applications of the two-stage concept for the collimation of ion beams. Most of the ion collimation studies were done for CERN LHC lead beam operation, where the specific problem is fragmentation and large momentum losses of the halo particles in the primary collimator $[25,26]$. Besides the conventional collimation concept used in BNL RHIC, a novel bent crystal channeling technique $[27,28]$ was tested for proton and heavy ion beams [29]. This method is based on deflection of the halo particles using a bent crystal as the primary collimator, and it is also being developed for the collimation of proton and ion beams at CERN [30,31]. Another advanced technique is the collimation using a hollow electron beam $[32,33]$. This concept is being developed at Fermilab and CERN and might overcome the problem of the ion fragmentation in the primary collimator [32]. 
The SIS100 collimation concept for partially stripped ion beams relies on the change of the charge state of the halo particles using a stripping foil. Consequently, the stripped ions can be deflected towards a dump location by using a beam optical element (e.g., a quadrupole magnet). This concept is similar to the collimation concept for ${ }^{1} \mathrm{H}^{-}$beams $[34,35]$. It should be mentioned that in the SIS100 there is an additional collimation system which was designed for partially stripped ion beams. It consists of sixty "cryocatchers" located in the cold arcs [36]. The purpose of this system is to collimate beam ions lost due to charge exchange as a result of their interaction with residual gas molecules [36,37].

The structure of the paper is as follows: Sec. II deals with the short description of the two-stage betatron collimation system. In Sec. III, interaction processes of ions with a thin foil (primary collimator) are analyzed. The design of the SIS100 collimation system for fully stripped ions, particletracking simulations, and calculation of the collimation efficiency are presented in Sec. IV. In this section also an effect of imperfections, errors, and beam parameters on the collimation efficiency is treated. The concept for the collimation of partially stripped ions in SIS100 is described in Sec. V. The summary and conclusions are given in Sec. VI.

\section{TWO-STAGE BETATRON COLLIMATION DESIGN}

Detailed beam optics specifications of the two-stage betatron collimation system in 1D and 2D are derived in Refs. [11,12,21]. The two-stage system consists of (a) a primary collimator (a thin foil) which intercepts and scatters the halo particles and (b) secondary collimators (bulky blocks) which are needed to absorb the scattered particles. Halo particles have a small impact parameter on the primary collimator. The impact parameter is a transverse distance from the edge of the collimator to the impact point of the halo particle. The impact parameter on the secondary collimator is enlarged due to scattering. The secondary collimators are located farther from the beam envelope than the primary collimator by a "retraction distance":

$$
\vartheta=\frac{n_{S}}{n_{P}}-1,
$$

where $n_{P}$ and $n_{S}$ are the normalized apertures of the primary and secondary collimators, respectively. Optimal phase advances for maximum collimation efficiency for given values of $n_{P}$ and $n_{S}$ can be calculated by using the formulas

$$
\begin{aligned}
& \mu_{S 1}=\arccos \left(\frac{n_{P}}{n_{S}}\right), \\
& \mu_{S 2}=\pi-\mu_{S 1},
\end{aligned}
$$

where $\mu_{S 1}$ and $\mu_{S 2}$ are the phase advances between the primary-1st secondary and the primary-2nd secondary collimator, respectively $[11,12,21]$.

On the basis of the theoretical background presented in Refs. [11,12,21], the collimation efficiency can be expressed as the ratio of the particles intercepted by the secondary collimators (collimated particles) to the particles scattered before in the primary collimator. This is the definition of the single-pass collimation efficiency, where the particles are tracked only from the primary up to the 2nd secondary collimator $[11,12,38]$. However, we consider the motion in circular accelerators (synchrotrons). Then the particles which are scattered at a small angle in the primary collimator and are not intercepted immediately by the secondary collimators can be still collimated in the next turns [13-21].

The multipass collimation efficiency is defined from the multiturn particle motion. It is expressed as the ratio of the number of collimated particles to the total amount of beam losses (collimated particles + particles intercepted by other lattice elements). For the calculation of the multipass collimation efficiency, a detailed particle tracking and the estimation of the beam loss distribution using simulation codes is necessary [13-21]. The simulations and calculation of the multipass collimation efficiency were performed for SIS100 and are presented in Sec. IV.

For the collimation design, two simulation tools of different types are needed to perform required calculations: (a) a code which is able to simulate the physical processes of the particle interaction with the collimator material (e.g., FLUKA) and (b) a code which can track particles through the accelerator lattice (e.g., MAD-X). FLUKA is a multipurpose Monte Carlo software package for simulation of interaction and transport of particles in matter [39-41]. MAD-X is a computer code developed for accelerator design, beam optics optimization, particle tracking, and beam dynamics simulation $[42,43]$.

The interaction of halo particles with the scattering foil (primary collimator) and its dependence on the primary ion species is an important issue for the performance of the collimation system. The interaction processes were studied for a $1 \mathrm{~mm}$ thick tungsten foil chosen as the primary collimator in SIS100. The thickness and material of the primary collimator are identical or close to those used in other synchrotrons of similar beam parameters [16-23].

Secondary collimators in this study are assumed to be ideal (black) absorbers. This means that no particles escape from the secondary collimator jaw. In reality, the collimated primary particles undergo nuclear interactions with the collimator material. It results in a shower of secondary particles mainly through spallation reactions. They have to be treated by using additional shielding around and particularly downstream of the collimator blocks. Heavy ions, especially at low energies, can even lose the total kinetic energy in the secondary collimator just via 
electronic stopping without nuclear interaction [5]. Interaction of the primary and production of the secondary particles, their fluence, and spatial distribution can be simulated by using FLUKA. The simulation data can be then utilized for the design of the shielding parameters. However, this subject is out of the scope of this paper.

The main goal is to find out if the collimation system originally designed for protons can be used also for the fully stripped ions. In order to do this, the following processes and their dependence on the ion species must be investigated: (i) scattering of the halo particles in the primary collimator, (ii) momentum (energy) losses of the halo particles in the primary collimator, and (iii) inelastic nuclear interaction of the halo particles with the collimator material, which for the ions means mainly hadronic fragmentation and electromagnetic dissociation (EMD) through the strong nuclear and electromagnetic force, respectively.

All these processes have a direct impact on the collimation efficiency. It is reasonable to perform the calculations for some reference physical quantity related to the beam parameters which is in this case magnetic rigidity $B \rho$. Magnetic rigidity was chosen as the reference quantity because it determines the injection and extraction energy of the beam.

\section{ION-FOIL INTERACTION PROCESSES}

\section{A. Scattering of the halo particles in the primary collimator}

The scattering process of the halo particles was simulated by using FLUKA. The model implemented in the code is based on the Molière theory of multiple Coulomb scattering improved by Bethe $[39,40,44]$. Besides the multiple Coulomb scattering, FLUKA is able to simulate also the single scattering which is based on the Rutherford formula. The single scattering was activated at the boundary crossing or when the validity conditions of Molière's theory for a current step are not satisfied. Nuclear form factors and spin-relativistic corrections were also involved in the simulation. For protons, FLUKA takes into account also the elastic nuclear scattering using tabulated data for the cross-section calculation $[39,40]$. The angular distribution was calculated for various ion species from proton up to uranium scattered by a $1 \mathrm{~mm}$ thick primary collimator made of tungsten. The dependence of the rms value of the projected deflection angles, $\theta_{\mathrm{rms}}$, on the magnetic rigidity is presented in Fig. 1.

One can observe that at low rigidities $\theta_{\text {rms }}$ for the heavier ions is larger than for protons. Towards the higher rigidities $\theta_{\text {rms }}$ for protons and heavier ions starts to coincide, and above $20 \mathrm{Tm}$ the values are practically the same. Already at the SIS18 maximum rigidity $(18 \mathrm{Tm})$, the $\theta_{\text {rms }}$ values are very similar for all considered ion species. This is due to the fact that momentum $p$ for various ion species at the same

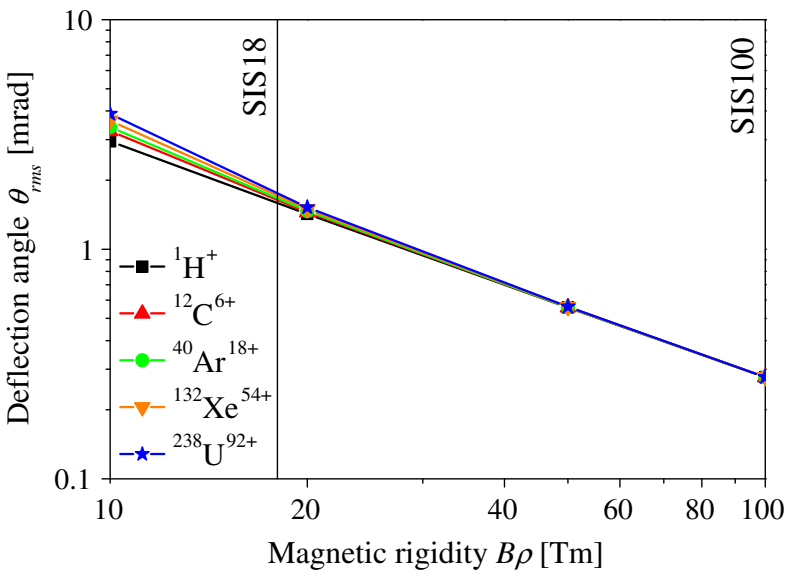

FIG. 1. Dependence of the projected deflection angle $\theta_{\text {rms }}$ on the magnetic rigidity $B \rho$ for the particles scattered in the $1 \mathrm{~mm}$ thick primary collimator made of tungsten calculated by using FLUKA.

magnetic rigidity is increasing with the charge $q$ of the particle $(q=\mathrm{Ze}$, where $Z$ is the atomic number of the primary ion and $e$ is the elementary charge). However, $p$ is also in the denominator of the formula for calculation of the $\theta_{\mathrm{rms}}$, which is also proportional to the $Z$ of the particle [44]. The angle $\theta_{\mathrm{rms}}$ is larger for heavier projectiles at lower rigidities due to the smaller $\beta$ relativistic parameter, which is at high rigidities close to 1 .

\section{B. Momentum losses of the halo particles in the primary collimator}

The energy and consequently the momentum losses of the halo particles were calculated by using FLUKA. The stopping power of charged particles in FLUKA is calculated by using the Bethe formula $[39,40,44]$. There are also higher order corrections implemented: Barkas correction, Bloch correction, Mott corrections, and effective charge parameterization. Besides the particle interaction with atomic electrons, the nuclear stopping power is also included in FLUKA [41]. The mean relative momentum losses $\delta=-d p / p$ in $1 \mathrm{~mm}$ thick tungsten foil for various projectiles calculated by using FLUKA are presented in Fig. 2. The losses vary from $4.4 \times 10^{-4}$ for protons, through $8.2 \times 10^{-3}$ for ${ }^{40} \mathrm{Ar}^{18+}$, up to $4.9 \times 10^{-2}$ for ${ }^{238} \mathrm{U}^{92+}$ ions at injection energy (18 Tm).

\section{Inelastic nuclear interaction of the halo particles with the primary collimator}

The probability $P_{\text {in }}$ for the inelastic nuclear interaction (hadronic fragmentation and EMD) of the halo particles with the primary collimator material was calculated by using FLUKA. Cross sections for the inelastic nuclear interaction of protons are in FLUKA calculated by using a mixture of tabulated data and parameterized fits $[39,40]$. Cross sections for the hadronic fragmentation of ions are 


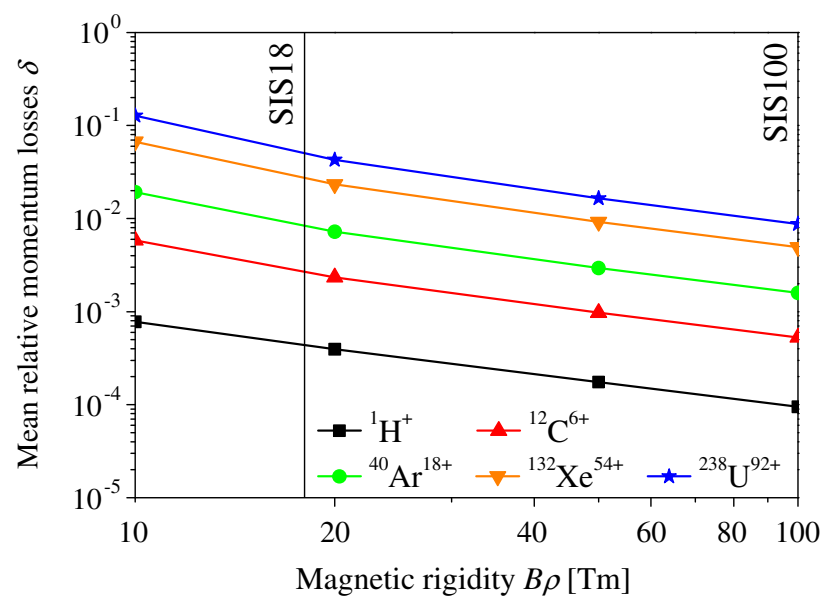

FIG. 2. Mean relative momentum losses in $1 \mathrm{~mm}$ thick primary collimator made of tungsten calculated by using FLUKA.

parametrized through the dual parton model by using Glauber formalism for energies $1 \mathrm{GeV} / \mathrm{u}$ and higher. At lower energies, Tripathi's cross-section parametrization is adopted $[45,46]$. The EMD process was also activated in the simulation for primary ions as well as for target nuclei. It is crucial especially for heavy ions [47-49]. Calculation of the EMD process cross section includes the equivalent photon spectrum and the cross section for the photonuclear reaction. The photonuclear reaction cross sections are compiled by using available databases based on the fitted measured values [49].

The $P_{i n}$ for various primary ions with magnetic rigidity 18 and $100 \mathrm{Tm}$, pertaining to injection and extraction in SIS100, are presented in Table I. In the case of ${ }^{1} \mathrm{H}^{+}$the probabilities for 18 and $100 \mathrm{Tm}$ are identical. The $P_{i n}$ shows a substantial increase with increasing mass and atomic number of the primary ions. A higher growth rate of the $P_{\text {in }}$ is observed at $100 \mathrm{Tm}$. As an example, for ${ }^{238} \mathrm{U}^{92+}$ ions at $100 \mathrm{Tm}$, the $P_{\text {in }}$ is more than twice higher compared to the $P_{\text {in }}$ at $18 \mathrm{Tm}$. The reason is that the cross section of the EMD process significantly increases with increasing atomic number of the primary ions and target nuclei. And it also greatly increases with increasing energy of the primary ions [47-49].

TABLE I. Probability $P_{\text {in }}$ for the inelastic nuclear interaction of the halo particles with $1 \mathrm{~mm}$ thick primary collimator made of tungsten calculated by using FLUKA.

\begin{tabular}{lcc}
\hline \hline & Probability $P_{\text {in }}$ & \\
\hline Beam & $18 \mathrm{Tm}$ & $100 \mathrm{Tm}$ \\
\hline${ }^{1} \mathrm{H}^{+}$ & 0.011 & 0.011 \\
${ }^{12} \mathrm{C}^{6+}$ & 0.022 & 0.023 \\
${ }^{40} \mathrm{Ar}^{18+}$ & 0.032 & 0.044 \\
${ }^{132} \mathrm{Xe}^{54+}$ & 0.067 & 0.117 \\
${ }^{238} \mathrm{U}^{92+}$ & 0.114 & 0.236 \\
\hline \hline
\end{tabular}

As a consequence of the hadronic fragmentation, primary ions usually break up into several secondary products with various masses [39-41]. The EMD process usually results in single or few nucleon ejection from the primary ion [47-49]. Motion of the fragments in the magnetic field is different from the primary ions due to the changed $A / Z$ ratio and momentum losses. Also, the standard deviation of the angular distribution is for the fragments higher than for the original primary particles. This is true as well for the protons interacting with the primary collimator via inelastic nuclear interactions. However, the heavy fragments coming from the EMD process can have the magnetic rigidity very close to the primary ions, especially if only a single nucleon is ejected. Also, their angular distribution and consequently the single-pass efficiency are similar to those of the primary ions [48].

\section{Selection of the material for the primary collimator}

The deflection angle $\theta_{\text {rms }}$, the probability of inelastic interaction $P$, and the momentum losses $\delta$ were analyzed for the purpose of the proper selection of the primary collimator material. The quantities were calculated for graphite, copper, and tungsten, which represent low, medium, and high $Z$ and density materials, respectively. The results for ${ }^{40} \mathrm{Ar}^{18+}$ ions at $18 \mathrm{Tm}$ calculated by using FLUKA are summarized in Table II.

It can be seen that, in order to reach the same $\theta_{\text {rms }}$ for all three materials, the $L$ is increasing by a factor of about 4 for copper and a factor of more than 50 for graphite in comparison with tungsten. An advantage of the thin primary collimator is a higher chance that a particle with a small impact parameter passes through its whole thickness. The $P$ is higher by a factor of about 3 and 18 for copper and graphite, respectively. The $\delta$ is higher by a factor of about 2.5 and 9 for copper and graphite, respectively. These results clearly indicate that high $Z$ construction materials are preferred for the primary collimator. On the other hand, for very high intensity and high power beams, radiation damage can play a crucial role in the selection of the collimator material. In this case, low $Z$ materials with very high radiation hardness and thermal stability, such as graphite or carbon composites, have to be used [13-15].

TABLE II. Deflection angle $\theta_{\text {rms }}$, thickness $L$, probability of nuclear interaction $P$, and momentum losses $\delta$ calculated for ${ }^{40} \mathrm{Ar}^{18+}$ ions at $18 \mathrm{Tm}$ in the primary collimator made of graphite, copper, and tungsten.

\begin{tabular}{lccc}
\hline \hline Material & Graphite & Copper & Tungsten \\
\hline$\theta_{\text {rms }}[\mathrm{mrad}]$ & 1.65 & 1.65 & 1.65 \\
$L[\mathrm{~mm}]$ & 51.8 & 4.1 & 1.0 \\
$P$ & 0.571 & 0.098 & 0.032 \\
$\delta$ & 0.075 & 0.019 & 0.008 \\
\hline \hline
\end{tabular}


The thermomechanical stability of tungsten as a construction material for the primary collimator in SIS100 was analyzed by using FLUKA simulation. The energy loss of a particle in a material $d E / d x$ is proportional to the square of its charge number (Bethe formula) [44]. But the total energy deposition depends also on the beam intensity. The energy deposition in tungsten was calculated for ${ }^{1} \mathrm{H}^{+},{ }^{40} \mathrm{Ar}^{18+}$, and ${ }^{238} \mathrm{U}^{92+}$ beams with the designed intensity $2 \times 10^{13}$, $1 \times 10^{11}$, and $1 \times 10^{10}$ particles per cycle, respectively [10].

The primary collimator jaw can be damaged due to fast beam losses over a short time period caused by beam instabilities or machine malfunction. We assume that such losses are intercepted by the edge of one primary collimator jaw (tungsten foil). The lost particles can have different transverse speeds and consequently different impact parameters (typically on the order of microns). These parameters are inversely proportional to the period of time $\tau$ during which the particles are lost (a period of time during which a significant decrease of the beam intensity is observed, typically on the order of milliseconds). When the transverse speed and impact parameters of the lost particles go down, $\tau$ goes up, and vice versa.

The simulation model is presented in Fig. 3. The impact area of the lost particles is $1 \mathrm{~cm}$ wide, which corresponds to the size of the beam. The particles are assumed to be uniformly distributed in the area with various impact parameters, defined by the maximum value $l_{\max }$. The transverse speed of the lost particles and consequently $l_{\max }$ for certain $\tau$ were calculated according to parameters of the SIS100 synchrotron, collimation system, and accelerated beam. It is approximately equal to $(200 \mu \mathrm{m} \mathrm{ms}) / \tau$. This means, for example, that $l_{\max }=200 \mu \mathrm{m}$ for $\tau=1 \mathrm{~ms}$ or $l_{\max }=20 \mu \mathrm{m}$ for $\tau=10 \mathrm{~ms}$.

Considering $10 \%$ of the beam intensity is lost in $\tau=$ $1 \mathrm{~ms}$ and $l_{\max }=200 \mu \mathrm{m}$, the energy deposition calculated by using FLUKA was 24,29 , and $79 \mathrm{~J} / \mathrm{g}$ for ${ }^{1} \mathrm{H}^{+},{ }^{40} \mathrm{Ar}^{18+}$, and ${ }^{238} \mathrm{U}^{92+}$ at $18 \mathrm{Tm}$, respectively. The difference between the energy deposition at 18 and $100 \mathrm{Tm}$ is within 5\%. The temperature rise was then obtained from the energy deposition using the specific heat capacity $c_{p}$, which is

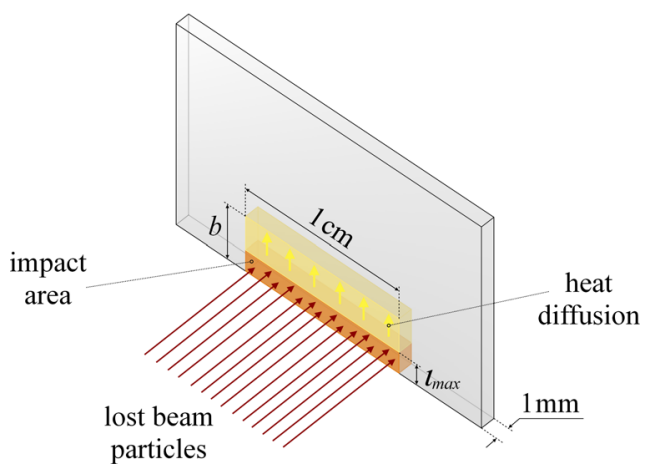

FIG. 3. Simulation model for the thermomechanical analysis of the primary collimator.
$0.133 \mathrm{~J} /(\mathrm{g} \mathrm{K})$ for tungsten at room temperature [50]. This is a conservative approach when we neglect any heat diffusion. The temperature rise for the considered beam losses is then 180,218 , and $594 \mathrm{~K}$ for ${ }^{1} \mathrm{H}^{+},{ }^{40} \mathrm{Ar}^{18+}$, and ${ }^{238} \mathrm{U}^{92+}$ beams, respectively. The melting point of tungsten is $3695 \mathrm{~K}$ [50].

When $\tau$ goes up and $l_{\max }$ goes down, the energy deposition is increasing for the same amount of losses. However, with increasing $\tau$ the heat diffusion starts to play an important role. The speed of heat diffusion is determined by the thermal diffusivity $\alpha$ of the material. $\alpha=\lambda /\left(\rho_{D} c_{p}\right)$, where $\lambda$ is the thermal conductivity and $\rho_{D}$ is the density of the material, for tungsten $177 \mathrm{~W} /(\mathrm{mK})$ at room temperature and $19.25 \mathrm{~g} / \mathrm{cm}^{3}$, respectively [50]. The $\alpha$ is then for tungsten equal to $69 \mathrm{~mm}^{2} / \mathrm{s}$.

We assume that the heat is through the foil diffused, in particular from the edge upwards, perpendicular to the direction of the particle motion (see Fig. 3). We can then reduce our problem to one dimension only (down-up) and calculate the thermal diffusion time as $t_{d}=b^{2} / \lambda$. This is the characteristic time which is required to reach a uniform temperature in a region with the relevant dimension $b$. For $b=200 \mu \mathrm{m}, t_{d}=0.58 \mathrm{~ms}$, which is shorter than $\tau$ associated with $l_{\max }$ of the same dimension $(200 \mu \mathrm{m}$ for $1 \mathrm{~ms})$.

Because of heat diffusion, in the case of $\tau$ longer than $1 \mathrm{~ms}$ and thereby $l_{\max }$ smaller than $200 \mu \mathrm{m}$, the impact area will not reach a critical temperature for a given amount of losses. On the contrary, for $\tau$ shorter than $1 \mathrm{~ms}$, the $l_{\max }$ increases and the temperature rise is lower than for $l_{\max }=200 \mu \mathrm{m}$. The $\lambda$ and $c_{p}$ are significantly temperature-dependent quantities [50], and $500 \mathrm{~K}$ above room temperature $\alpha=46 \mathrm{~mm}^{2} / \mathrm{s}$. The $t_{d}$ for $b=200 \mu \mathrm{m}$ then goes up to $0.87 \mathrm{~ms}$, which is still sufficiently low.

On the basis of the calculation, we can conclude that tungsten can be used as a construction material for the collimators in SIS100. The presented results indicate that a tungsten primary collimator can withstand even significantly higher losses than just $10 \%$. A more detailed analysis using specific simulation codes might be required, especially in the case of the external cooling system.

\section{HALO COLLIMATION OF FULLY STRIPPED IONS IN SIS100}

\section{A. Collimation system design}

The design of the collimation system for protons and fully stripped ions in SIS100 consists of one primary and two secondary stages (see Fig. 4). SIS100 will have a

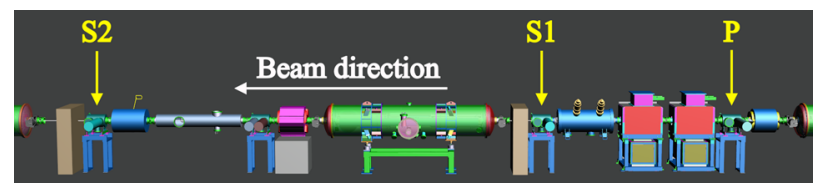

FIG. 4. Location of the collimation system in SIS100. 
hexagonal shape, and the collimators will be located in a straight section of sector 1 . The primary as well as the secondary collimators are designed to have a rectangular aperture, which means two jaws in the horizontal (right, left) and two jaws in the vertical (upper, lower) plane at each stage. The primary collimator jaws are assumed to be a $1 \mathrm{~mm}$ thick tungsten foils. The secondary collimator jaws are assumed to be $40 \mathrm{~cm}$ long also made of tungsten. The distance of the 1st and 2nd secondary collimator from the primary one is about 5 and $17 \mathrm{~m}$, respectively. The collimator jaws are assumed to be movable in the transverse direction by using a stepping motor in order to adjust the system for different lattice and beam parameters.

The optics of the 2D collimation system with a rectangular aperture cannot be simplified to two uncoupled horizontal and vertical 1D systems. It is due to the fact that the deflection angles of the particles after scattering in the primary collimator are equally distributed in both transverse planes [11,12]. For example, a particle which oscillates in the horizontal transverse plane can be scattered in the vertical direction. Since the 2D collimation in the horizontal and vertical transverse plane is not independent, the optimal phase advances between the primary and secondary stages for a 1D system (see Sec. II) cannot be applied. However, it is still possible to reach sufficiently high efficiency as a result of multiple transitions through the collimation system [11-23]. The particles which escape the collimation system can still be collimated after several turns in the synchrotron. To evaluate the overall performance of the collimation system, multiturn particletracking simulations are needed.

The absolute values of the beam emittance $\varepsilon_{x}$ and $\varepsilon_{y}$ in horizontal and vertical phase space, respectively, are slightly different for proton and ion beams. The beam in SIS100 will have an elliptical shape, and $\varepsilon_{x}$ is roughly 3 times larger than $\varepsilon_{y}$. The $\varepsilon_{x}$ and $\varepsilon_{y}$ of the proton beam are about 13 and $4 \mathrm{~mm} \times \mathrm{mrad}$, respectively. The $\varepsilon_{x}$ and $\varepsilon_{y}$ of the fully stripped ion beams are about 9 and $3 \mathrm{~mm} \times \mathrm{mrad}$, respectively. These numbers represent $2 \sigma$ of the beam distribution. The horizontal and vertical acceptance of the primary collimator jaws $\varepsilon_{A x}$ and $\varepsilon_{A y}$, respectively, are also different for proton and ion operation. The $\varepsilon_{A x}$ and $\varepsilon_{A y}$ for the proton operation are fixed at 65 and $20 \mathrm{~mm} \times \mathrm{mrad}$, respectively. The $\varepsilon_{A x}$ and $\varepsilon_{A y}$ for the ion operation are fixed at 40 and $15 \mathrm{~mm} \times \mathrm{mrad}$, respectively. These values correspond to $4.2-4.5 \sigma$ of the beam distribution. The retraction distance $\vartheta$ was chosen to be 0.1 for all primary ion species, which represents the absolute value about $2 \mathrm{~mm}$.

SIS100 will operate with at least three different working points [10]. The first one is proposed for the proton operation, the second and the third ones for the ion operation in the fast and slow extraction regime, respectively (see Table III). The reference working points for the same operation modes were used also in the presented
TABLE III. Horizontal $\left(Q_{x}\right)$ and vertical $\left(Q_{y}\right)$ tune for proton and ion operation in SIS100.

\begin{tabular}{lcc}
\hline \hline Operation mode & $Q_{x}$ & $Q_{y}$ \\
\hline Proton & 21.8 & 17.7 \\
Ion (fast extraction) & 18.88 & 18.8 \\
Ion (slow extraction) & 17.31 & 17.8 \\
\hline \hline
\end{tabular}

collimation study. They can, of course, differ from the tunes chosen for the actual operation in SIS100. As an example, the beta and dispersion functions of the SIS100 lattice for the ion operation in the fast extraction mode are presented in Fig. 5.

\section{B. Particle-tracking simulations}

The particle-tracking simulations were performed by using MAD-X $[42,43]$. For the transversal motion of the halo particles, a diffusion rate model was adopted [51,52]. This model is based on a constant slow diffusion of the halo particles towards the aperture restriction of the accelerator lattice, which is usually of the order of a micron per turn and less [51,52]. We assume that the halo particles are by definition intercepted by the primary collimator jaw as an acceptance limit (in $\mathrm{mm}$ mrad) of the SIS100 with a small impact parameter. The impact parameter is a transverse offset between the impact point of the halo particles and the edge of the primary collimator jaw. It depends on the diffusion rate and therefore varies from tenths of a nanometer up to a few microns [51,52].

The interaction of the halo particles with the primary collimator jaws was simulated for all ions by using FLUKA. We assume a uniform distribution of the halo particles on the collimator jaws in the horizontal and vertical directions along the inner edge of the rectangular aperture. For the particle tracking, the thin lens module of MAD-X was used.

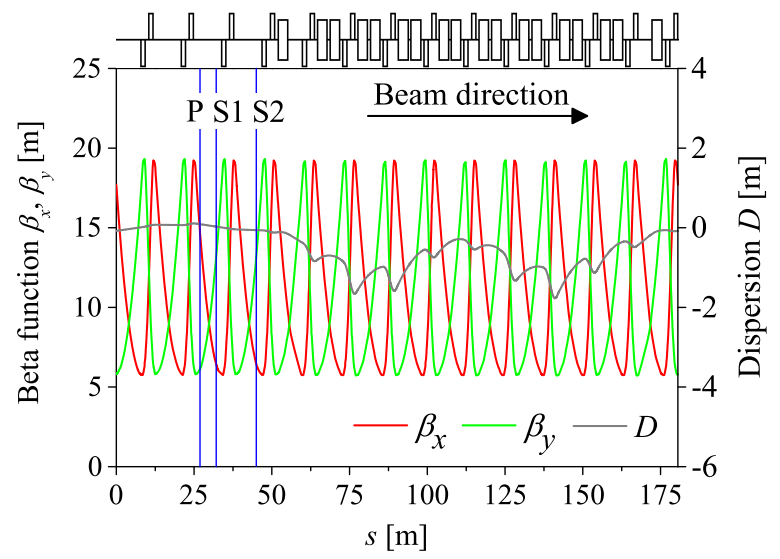

FIG. 5. Beta $\beta_{x}$ and $\beta_{y}$ and dispersion $D$ functions of one superperiod of the SIS100 lattice for the ion operation in the fast extraction mode with the marked position of the primary and secondary collimators. 
Simulation tools for the interaction with the primary collimator and particle tracking were linked "offline." Firsy, the scattering and momentum losses in the primary collimator were simulated by using FLUKA. Consequently, a set of particles with an angular distribution and momentum deviation (change of the rigidity) after the interaction with the foil was generated. These data were used as an input to the MAD-X simulation.

The particles were then tracked starting from the position of the primary collimator until they are intercepted either by the secondary collimators or by other elements of the SIS100 lattice. The initial transverse position of the tracked particles is equal to the aperture of the primary collimator. Taking into account the impact parameter up to a few microns did not result in any difference in the estimated beam loss distributions. A fraction of the particles can hit the primary collimator again after several turns. In this case, their transverse coordinates (positions and angles) were there recorded. For these coordinates the interaction of the particles with the primary collimator was again simulated by using FLUKA. After the calculation of the additional angular kick and momentum loss, the particle tracking using MAD-X continued. This procedure was repeated by using simple scripts until all of the simulated particles are either collimated or lost somewhere in the accelerator lattice. Aperture limits of each lattice element were defined in the simulation model.

As an example, the distribution of the lost beam particles for ${ }^{1} \mathrm{H}^{+},{ }^{40} \mathrm{Ar}^{18+}$, and ${ }^{238} \mathrm{U}^{92+}$ along the SIS100 lattice are shown in Fig. 6. It can be seen that nearly all losses of the proton beam are intercepted by the secondary collimators. In the case of the ${ }^{40} \mathrm{Ar}^{18+}$ beam, a fairly substantial fraction of the halo particles is collimated by the cryocatchers. And for the ${ }^{238} \mathrm{U}^{92+}$ beam, a significant number of the loss particles end up on a few cryocatchers. The cryocatchers are a part of the combined collimation and pumping system designed for interception of the partially stripped ions which interact with the residual gas molecules [36].

The multiturn particle-tracking simulations were performed only for the primary particles. This means that the presented beam loss maps and calculated collimation efficiencies do not include secondary particles produced through the inelastic nuclear interaction in the primary collimator. The production and detailed tracking of the secondary particles generated in the primary as well as in the secondary collimators is an important topic of further studies. Nevertheless, the single-pass collimation efficiency of the secondary particles was estimated (see Sec. IV C).

\section{Efficiency of the collimation system}

The multipass collimation efficiency has already been defined as the ratio of the number of collimated particles to the total amount of beam losses (see Sec. IID). The collimation efficiency in SIS100 was calculated for various primary beams from ${ }^{1} \mathrm{H}^{+}$up to ${ }^{238} \mathrm{U}^{92+}$. The results of the
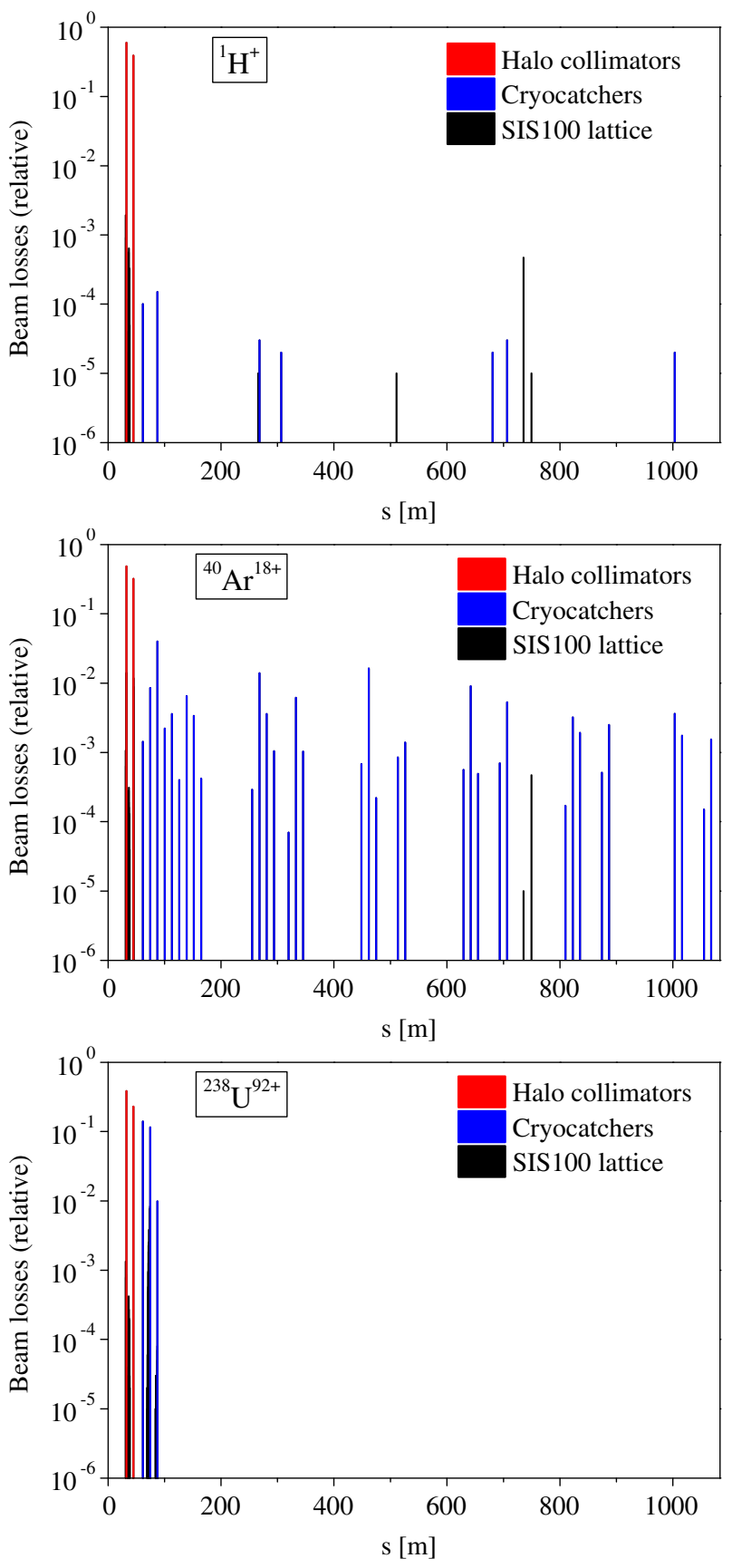

FIG. 6. Distribution of the lost beam particles along the SIS100 lattice for ${ }^{1} \mathrm{H}^{+},{ }^{40} \mathrm{Ar}^{18+}$, and ${ }^{238} \mathrm{U}^{92+}$ beams. The ion-foil interaction and the particle tracking were simulated by using FLUKA and MAD-X, respectively.

calculation for the fast extraction operation mode are presented in Fig. 7. The multipass efficiency is compared with the single-pass one. Besides the multipass efficiency of the halo collimators, also the contribution of the cryocatchers was taken into account. It was found out that almost all the halo particles are either collimated or lost in 100 turns after the first interaction with the primary collimator for all beam species. 


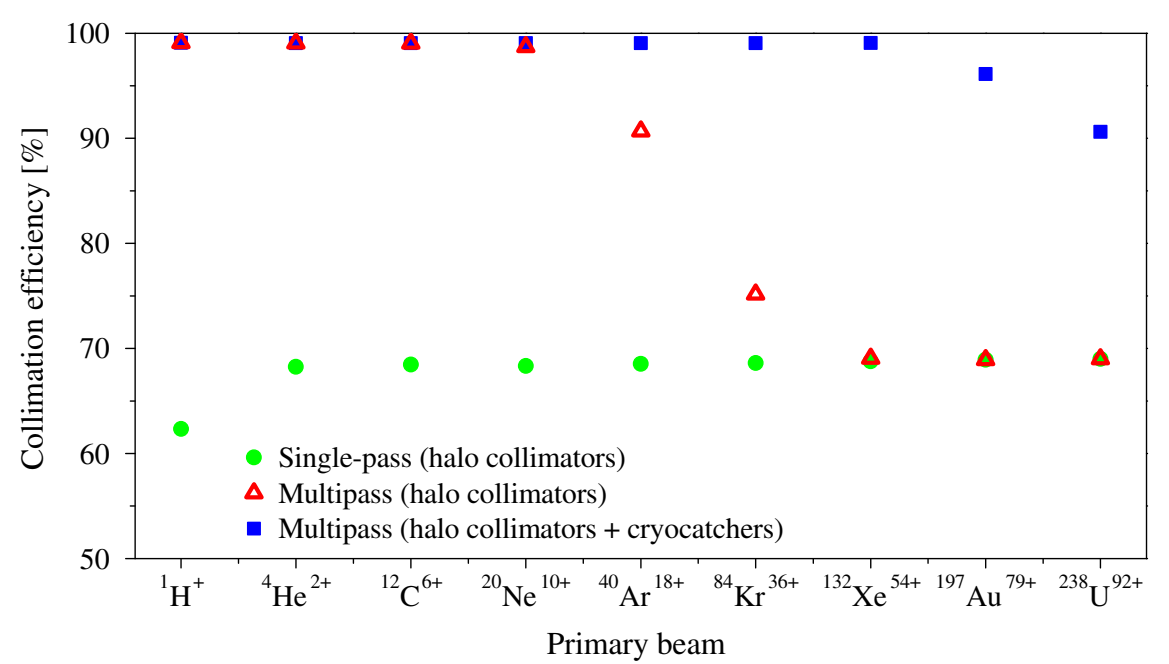

FIG. 7. Collimation efficiency calculated for various primary beams from proton up to uranium in SIS100.

The single-pass efficiency is lower for proton beams than for ion beams. This is caused by various factors: (i) different beam emittance and consequently the size of the beam, (ii) different working point for proton operation and shape of the beta functions, and (iii) smaller deflection angles due to scattering in the primary collimator (see Fig. 1). The beam size and the beam optics parameters affect the quantities important for the calculation of the single-pass efficiency. It is the absolute value of the retraction distance as well as the phase advance between the stages of the collimation system. The absolute retraction distance is larger for heavy ions than for light ions due to the larger beam size. The larger the retraction distance, the lower the single-pass efficiency.

The multipass efficiency without cryocatchers is about $99 \%$ from protons up to ${ }^{20} \mathrm{Ne}^{10+}$. It starts to decrease from ${ }^{40} \mathrm{Ar}^{18+}$, and it drops to the level of the single-pass efficiency at ${ }^{132} \mathrm{Xe}^{54+}$. This is due to the high momentum losses of heavy ions in the primary collimator in comparison with protons and light ions (see Sec. III B). Because of this fact, multiple transitions through the collimation system are not possible, and the particles which were not collimated are lost in the arc sections of SIS100. The multipass efficiency can be significantly improved with the help of the cryocatchers. Then the $99 \%$ level is reached up to ${ }^{132} \mathrm{Xe}^{54+}$, and even for ${ }^{238} \mathrm{U}^{92+}$ is the efficiency is about $90 \%$.

The collimation efficiency of the heavy ion beams was calculated also for the slow extraction operation mode. It was found that the differences in comparison with the fast extraction mode presented in Fig. 7 are within $2 \%$.

The calculated collimation efficiencies do not include the secondary particles produced through the inelastic nuclear interaction. However, their angular deflection downstream of the primary collimator was simulated by using FLUKA. The angular distribution of the secondary particles depends on the interaction process (hadronic fragmentation or
EMD). Products of the hadronic fragmentation undergo a larger angular deflection than the EMD products which have the angular distribution close to those of the primary ions. However, we can clearly say that in both cases the $\theta_{\text {rms }}$ of the secondary fragments is larger than for the primary particles. Their single-pass efficiency is also assumed to be higher than for the primary particles where it reaches almost $70 \%$ at $18 \mathrm{Tm}$ (injection energy). This number can be significantly lower in the case of higher beam rigidities (see Sec. IV E). On the other hand, most of the beam losses are expected at $18 \mathrm{Tm}$, where the emittance is the largest and the injection process fills up the longest time period of the cycle duration.

\section{Impact parameter and retraction distance}

The diffusion of the halo particles is a slow process, and therefore their impact parameter can be so small that particles do not pass through the full thickness of the primary collimator. For this reason, the impact parameter can have a significant influence on the particle interaction with the primary collimator jaw as well as on the singlepass efficiency (see Sec. III). The influence of the initial impact parameter on the multipass efficiency was estimated again by using the particle-tracking simulations (see Fig. 8). Only the halo collimators are included in the presented calculations. Taking into account the cryocatchers, almost no dependence of the collimation efficiency on the impact parameter was observed.

It was found out that most of the particles impinging on the primary collimator jaw with a small impact parameter are not intercepted directly by the secondary collimators. However, they hit the primary jaw again after several turns with a significantly larger impact parameter sufficient to pass through its full thickness. That is why for the light ions (e.g., ${ }^{12} \mathrm{C}^{6+}$ ) the impact parameter does not affect the multipass efficiency of the collimation system. 


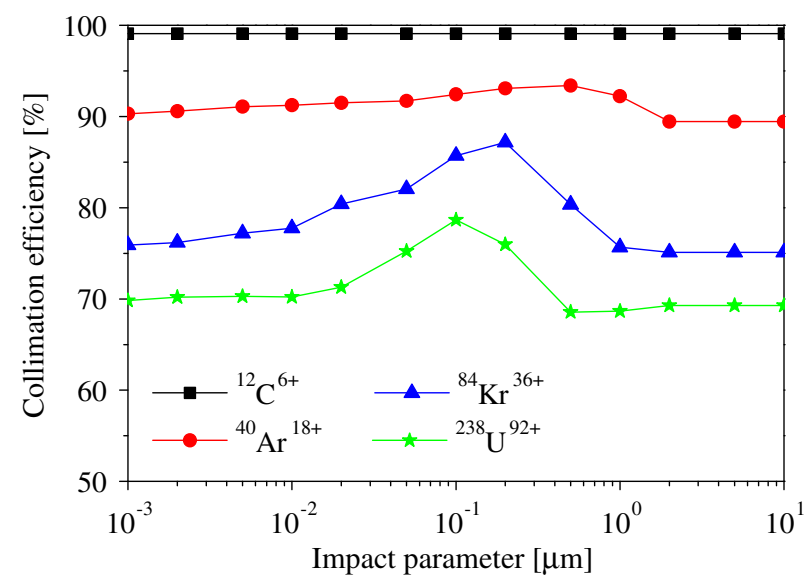

FIG. 8. Influence of the initial impact parameter on the multipass collimation efficiency of the halo collimation system (without cryocatchers).

For heavier ions (from ${ }^{40} \mathrm{Ar}^{18+}$ ), the situation is different due to the momentum losses in the primary collimator. When the impact parameter is very small (order of nanometers), there is almost no angular deflection and momentum losses in the primary collimator. The particles are neither intercepted by the secondary collimators nor lost in the arc sections. However, they hit the primary collimator again with a larger impact parameter. That is why the collimation efficiency is similar for the impact parameter below 0.01 and above $1 \mu \mathrm{m}$. When the impact parameter is between those two values, particles have also a small angular deflection and momentum losses, so they are neither collimated nor lost. However, for the impact parameters above $0.01 \mu \mathrm{m}$, the angular deflection is not negligible. It is an additional scattering for the particles when they hit the primary collimator again after several turns. There is then a higher probability to be intercepted by the secondary collimators. For this reason, the multipass collimation efficiency increases for the initial impact parameter between 0.01 and $1 \mu \mathrm{m}$ (see Fig. 8).

The collimation efficiency was also calculated for various retraction distances. Up to now, the calculations were performed only for the retraction distance 0.1 in the case of all primary beams. The exact values depend on the operation mode of SIS100 and on the size of each primary ion beam. The effect of the higher values of the retraction distance 0.2 and 0.3 on the collimation efficiency was investigated. The retraction distance values $0.1,0.2$, and 0.3 represent about 2,4 , and $6 \mathrm{~mm}$, respectively. The multipass collimation efficiency calculated without taking into account the cryocatchers is presented in Fig. 9.

In the case of the light ions like ${ }^{12} \mathrm{C}^{6+}$, the multipass efficiency varies only slightly with increasing retraction distance. However, for heavy projectiles like ${ }^{238} \mathrm{U}^{92+}$, there is an absence of the multiple transitions through the collimation system (see Fig. 7) due to the large momentum losses after interaction with the primary collimator (see

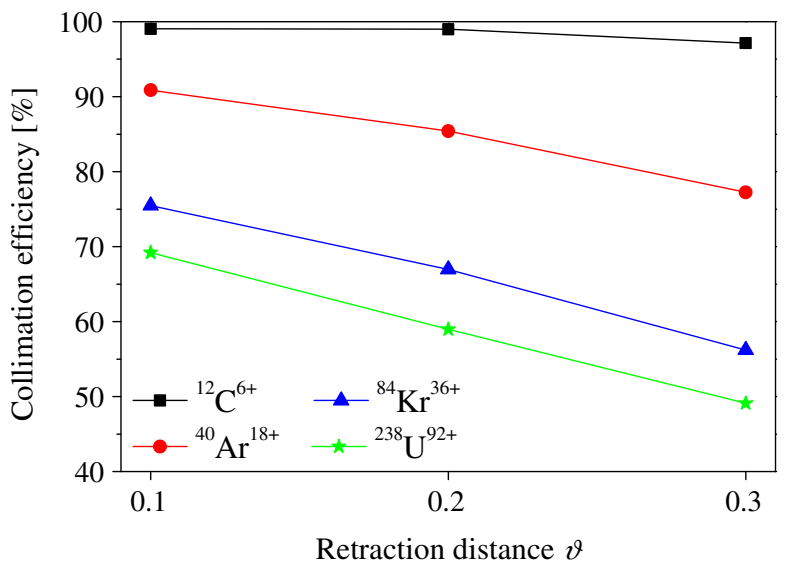

FIG. 9. Dependence of the collimation efficiency on the retraction distance.

Fig. 2). When the retraction distance is big, fewer particles are collimated in the single-pass process. The heavy ions which are not intercepted by the secondary collimators are then lost downstream of the collimation system. On the contrary, the light ions which escape in the first turn still have a chance to be collimated due to multiple transitions through the collimation system.

The difference in the multipass efficiency calculation with cryocatchers was less than $1 \%$ in the case of ${ }^{12} \mathrm{C}^{6+}$, ${ }^{40} \mathrm{Ar}^{18+}$, and ${ }^{84} \mathrm{Kr}^{36+}$ ions for all considered retraction distances. Bigger discrepancies were observed only in the case of the ${ }^{238} \mathrm{U}^{92+}$ ions. The collimation efficiency was reduced in comparison with the retraction distance 0.1 by about $3 \%$ and $5 \%$ for the retraction distances 0.2 and 0.3 , respectively.

The distribution of the halo particles on the primary collimator jaws was assumed to be uniform up to now. In addition, simulations with Gaussian and pointlike distribution for ${ }^{1} \mathrm{H}^{+},{ }^{40} \mathrm{Ar}^{18+}$, and ${ }^{238} \mathrm{U}^{92+}$ ions were performed. The Gaussian distribution was assumed to be truncated at $\pm 2 \sigma$ standard deviations, where the $2 \sigma$ represents the aperture of the primary collimator. The pointlike distribution is represented the following particle coordinates: if $x=A_{x}$, then $y=0$, and if $y=A_{y}$, then $x=0$. The $x$ and $y$ are the horizontal and vertical particle coordinates, respectively, and the $A_{x}$ and $A_{y}$ are the horizontal and vertical apertures of the primary collimator, respectively. For such distributions, the multipass collimation efficiency with and without cryocatchers was calculated. The biggest discrepancy was observed between the efficiencies calculated without cryocatchers using uniform and pointlike distributions of the ${ }^{238} \mathrm{U}^{92+}$ ions. The difference was less than $3 \%$ and in all other cases not more than $1 \%$. This is again caused by the fact that for the ${ }^{238} \mathrm{U}^{92+}$ there are no multiple transitions through the collimation system and the singlepass efficiency is more affected by the scattering in the primary collimator. 


\section{E. Closed orbit distortion and beam parameters}

The closed orbit distortion (COD), which is typically induced by misalignment of the magnets and field errors, was also taken into consideration. In order to get reasonable statistics, 100 different error seeds were simulated for the COD calculations using MAD-X. The radial COD rms values (root sum squares of the horizontal and vertical values) ranged from 1.3 up to $5.2 \mathrm{~mm}$. These numbers are considered as a conservative estimate for the corrected COD of SIS100 [53] in order to demonstrate the robustness of the collimation system. The collimation system was not readjusted according to the COD, and the jaws remain at the same transverse position for nearly all of the error seeds. Only in a few cases of the big COD values, where a secondary collimator becomes an acceptance limit instead of the primary one, was the system realigned. This will be possible also in reality, since the collimator jaws are designed to be movable by using stepping motors. Results of the multipass efficiency calculation without cryocatchers for various COD values are presented in Fig. 10.

For the calculated number of seeds, the mean value of the COD is bigger than $3 \mathrm{~mm}$. It was found out that for protons and very light ions there is a very small effect of the COD on the collimation efficiency. The COD starts to affect significantly the collimation efficiency of the ion beams above ${ }^{12} \mathrm{C}^{6+}$. It is caused by the bigger momentum losses of the heavier projectiles in the primary collimator. On the other hand, for the very heavy projectiles starting from ${ }^{132} \mathrm{Xe}^{54+}$, the momentum losses in the primary collimator are too big and multiple transitions through the collimation system are then not possible (see Fig. 7). For this reason, there is again almost no effect of the COD on the collimation efficiency above ${ }^{132} \mathrm{Xe}^{54+}$. For all simulated error seeds, the arithmetic mean value and the standard

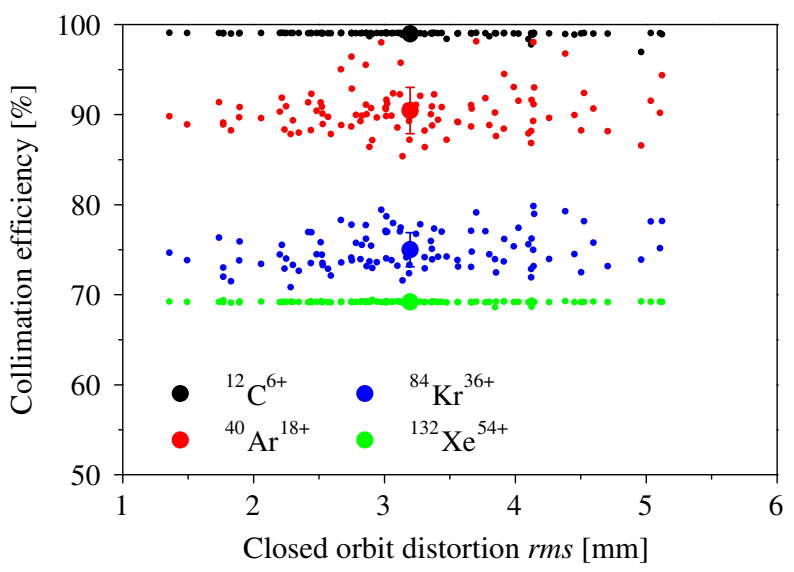

FIG. 10. Dependence of the multipass collimation efficiency without cryocatchers on the closed orbit distortion. The small circles are the individual error seeds. The big circles are the arithmetic mean values of the COD and efficiency. The error bars are the standard deviations for the efficiency. deviation $(1 \sigma)$ of the collimation efficiency were calculated. The maximum standard deviation was obtained in the case of ${ }^{40} \mathrm{Ar}^{18+}$ beam and represents $\pm 3 \%$ variation of the collimation efficiency. The influence of the COD on the multipass efficiency taking into account the cryocatchers was observed to be negligible.

The collimation efficiency was also calculated for higher energies corresponding to magnetic rigidities 50 (medium range energy of SIS100) and $100 \mathrm{Tm}$ (extraction energy of SIS100). The efficiency was again calculated for various primary beams from protons up to uranium ions. Results of the calculation are presented in Fig. 11. There is also a comparison of the single-pass, multipass, and multipass with cryocatchers efficiencies.

It can be seen that the single-pass efficiency decreases drastically with increasing energy of the primary ions. At extraction energy, the single-pass efficiency is almost negligible. The multipass efficiency is in the case of protons and ${ }^{12} \mathrm{C}^{6+}$ ions about $99 \%$. For the heavier ions it starts to decrease and drops to about $20 \%$ in the case of ${ }^{238} \mathrm{U}^{92+}$ at extraction energy. This was expected, since the deflection angle is decreasing with the increasing energy. However, taking into account the cryocatchers, the multipass collimation efficiency reaches the level of $99 \%$ for all primary ions.

It was observed before that for heavy ions at the injection energies the momentum losses in the primary collimator are too high (see Fig. 2). For this reason, a stable multiturn particle motion is not possible due to momentum acceptance of the synchrotron. However, at higher beam energies, differences between the single-pass and multipass efficiency are observed (see Fig. 11). They are caused by the fact that the absolute momentum losses in the primary collimator, $-d p$, differ at higher energies only slightly from the values at injection energies. On the other hand, the momentum $p$ is about 5 times larger at the extraction energies $(100 \mathrm{Tm})$ than at the injection energies $(18 \mathrm{Tm})$.

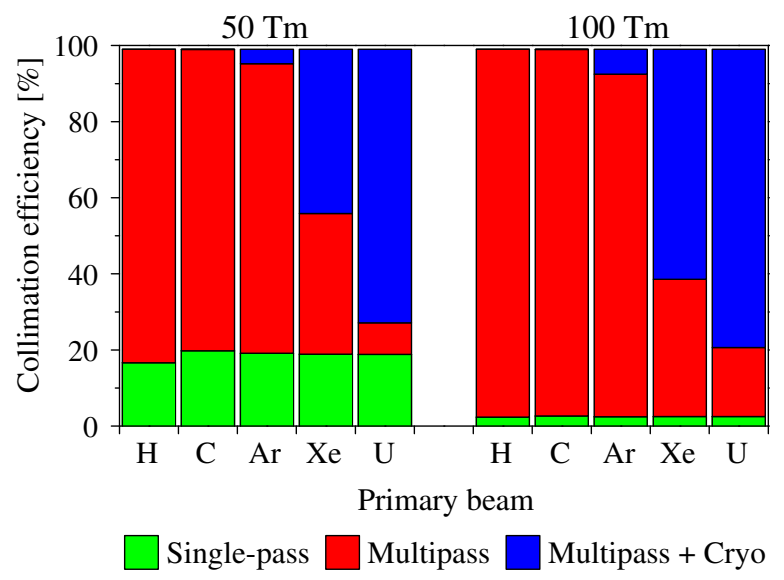

FIG. 11. Collimation efficiency of ${ }^{1} \mathrm{H}^{+},{ }^{12} \mathrm{C}^{6+},{ }^{40} \mathrm{Ar}^{18+}$, ${ }^{132} \mathrm{Xe}^{54+}$, and ${ }^{238} \mathrm{U}^{92+}$ primary beams at higher energies corresponding to the magnetic rigidities 50 and $100 \mathrm{Tm}$. 
That is why $\delta=-d p / p$ is decreasing with increasing energy, below the momentum acceptance. Then multiple transitions through the collimation system become possible for heavy ions.

The effect of the beam momentum spread $d p / p$ on the collimation efficiency was investigated for ${ }^{1} \mathrm{H}^{+}$, ${ }^{12} \mathrm{C}^{6+},{ }^{40} \mathrm{Ar}^{18+},{ }^{132} \mathrm{Xe}^{54+}$, and ${ }^{238} \mathrm{U}^{92+}$ primary ions. The distribution of the momentum spread was assumed to be Gaussian truncated at $\pm 2 \sigma$ standard deviations. Synchrotron motion was also included in the simulations. The collimation efficiency was calculated for three different values, namely, $1 \times 10^{-3}, 3 \times 10^{-3}$, and $5 \times 10^{-3}$, which represent the $2 \sigma$ of the momentum spread distribution.

Almost no effect of the considered momentum spread values on the collimation efficiency was observed. In the case of the multipass efficiency without cryocatchers, the difference in the calculated collimation efficiencies was less than $1 \%$ for all ion species. The impact of the different momentum spread values on the multipass efficiency with cryocatchers was observed to be utterly negligible.

\section{F. Tolerable beam losses- $1 \mathrm{~W} / \mathrm{m}$ criterion}

The beam loss maps were analyzed in order to estimate if the criteria for uncontrolled beam losses of $1 \mathrm{~W} / \mathrm{m}$ were fulfilled. We assume that due to low diffusion speed all of the halo particles first hit the primary collimator as an acceptance limit of the accelerator lattice and are considered to be lost. Consequently, most of them are collimated (intercepted by the secondary collimators). The collimated halo particles are lost in a controlled way (controlled losses). The halo particles which are not collimated but are intercepted by other lattice elements are assumed to be lost in an uncontrolled way (uncontrolled losses). The amount of the uncontrolled losses should be below $1 \mathrm{~W} / \mathrm{m}$. We have finally calculated a fraction (percentage) of the total beam intensity which can hit the primary collimator, and the uncontrolled losses are still kept below $1 \mathrm{~W} / \mathrm{m}$ in the whole machine. This fraction of the beam is specified as tolerable beam loss which can be handled by the collimation system to fulfill the criteria. The SIS100 design beam intensities varies from $2 \times 10^{13}$ particles per cycle for protons to $1 \times 10^{10}$ particles per cycle for ${ }^{238} \mathrm{U}^{92+}$ ions [10]. The length of the cycle for proton operation is $2.8 \mathrm{~s}$. For ion operation there are two regimes: slow extraction and fast extraction. An average length of the ion beams cycle is about $5 \mathrm{~s}$.

In the case of proton and very light ion beams, the highest local uncontrolled losses are observed close to the secondary collimators and at the slow extraction septa (see Fig. 6). The fraction of the design beam intensity which can hit the primary collimator so that the uncontrolled losses are still below $1 \mathrm{~W} / \mathrm{m}$ is about $10 \%$. For the medium weight ions like ${ }^{40} \mathrm{Ar}^{18+}$, the highest uncontrolled losses are observed near the cryocatchers (see Fig. 6). The tolerable beam losses are assumed to be about 5\%. The highest uncontrolled losses were observed in the case of ${ }^{238} \mathrm{U}^{92+}$ ions again near the cryocatchers. The tolerable beam losses are assumed to be only about $2 \%$ of the designed beam intensity.

Since the $1 \mathrm{~W} / \mathrm{m}$ criterion gives information about the residual activation, it is important to take into account whether the particles are lost in a hot spot or not. Except the halo collimators, the hot spots are, for example, extraction septa or cryocatchers because of the losses from other mechanisms. The highest uncontrolled losses outside of the hot spots were observed for the ${ }^{238} \mathrm{U}^{92+}$ beam. The tolerable beam losses are assumed to be about $9 \%$ of the design beam intensity. These numbers are considered to be within a safety margin required for the project. Products of the inelastic nuclear interaction in the primary collimator are not included in the calculation. As was mentioned before, the detailed particle tracking and loss distribution of the secondary particles is a subject of further studies.

It should be also mentioned that the beam loss criteria for heavy ions are less strict than for protons [5]. For example, the loss criterion for $1 \mathrm{GeV} / \mathrm{u}{ }^{238} \mathrm{U}^{92+}$ beams is $5 \mathrm{~W} / \mathrm{m}$. However, the beam loss criteria were estimated only from $200 \mathrm{MeV} / \mathrm{u}$ to $1 \mathrm{GeV} / \mathrm{u}$. Injection energies of all ions to be accelerated in SIS100 are above this range. The lowest is $1.35 \mathrm{GeV} / \mathrm{u}$. The criteria are also approaching the value $1 \mathrm{~W} / \mathrm{m}$ with increasing energy of the beam. For this reason, the calculations of the tolerable beam losses are based on the value $1 \mathrm{~W} / \mathrm{m}$ for all ions and beam energies as a conservative estimate.

\section{HALO COLLIMATION OF PARTIALLY STRIPPED IONS IN SIS100}

\section{A. Collimation concept}

In order to reach high intensities of heavy ion beams, SIS100 will be operated with partially stripped ions of intermediate charge states. For instance, heavy ions of the following charge states are intended for the operation in SIS100: ${ }^{238} \mathrm{U}^{28+},{ }^{197} \mathrm{Au}^{25+},{ }^{181} \mathrm{Ta}^{24+},{ }^{132} \mathrm{Xe}^{22+}$, or ${ }^{84} \mathrm{Kr}^{17+}$ [54]. The concept considered for the halo collimation of the partially stripped ions is rather simple. It is based on the change of the initial ion charge state using a stripping foil. The ions which interact with the stripping foil can consequently be deflected towards a dump location by using a beam optical element (dipole or quadrupole magnets). This is because they experience a stronger magnetic force than the primary ions with the initial charge state in the presence of the same field.

It was found that dipoles are not suitable for the deflection, because the ions with the change of the initial charge state are intercepted by the aperture restriction in the middle of the magnet. However, the ions can be properly deflected by quadrupole magnets. The collimation system for partially stripped ions is planned to be located in the slow extraction area of SIS100. 
During the slow extraction, a fraction of the partially stripped ions interact directly with the electrostatic septum wires. These ions are then assumed to change their initial charge state to be close to fully ionized due to the stripping process in the wires. It was shown in a previous study that most of them are then lost at the two quadrupole magnets (doublet) downstream of the slow extraction septum [55]. For this reason, the two quadrupoles originally designed as superconducting as all the rest in the SIS100 lattice were changed into normal conducting magnets. Also, the beam pipe of this quadrupole doublet was redesigned. This was decided in order to avoid quenches [55].

This fact can be utilized also for the halo collimation design of partially stripped ions. The primary collimator (a stripping foil) is placed upstream of the warm quadrupole doublet in the slow extraction area. The intermediate charge state heavy ions are stripped of their remaining electrons and consequently are deflected by the magnetic field of the two warm quadrupoles. Finally, the ions are intercepted by the secondary collimators situated near the warm quadrupoles.

\section{B. Charge state distribution}

The collimation concept of the partially stripped ions is based on the change of their initial charge state using a stripping foil. An important issue is to predict the charge state distribution of the primary ions which passed through the foil. A detailed theoretical and experimental study of the charge exchange of partially stripped heavy ions is presented in Ref. [56]. Described physical models are implemented in GLOBAL, which can be used for calculation of the charge state distribution of ions traversing solid and gaseous targets [56,57]. GLOBAL is applicable for the interaction of projectiles having the initial charge number equal or higher than $q_{\max }-28$, where $q_{\max }$ is the fully ionized state. The code takes into account also the energy losses of the particle passing through the target. The initial energy of the ions can be set from $30 \mathrm{MeV} / \mathrm{u}$ up to $2 \mathrm{GeV} / \mathrm{u}$. The user can choose various output options: (i) charge state distribution of the projectile at the exit of the target, (ii) equilibrium charge state distribution of the projectile for chosen material, and (iii) charge state evolution of the projectile throughout the target [57].

The charge state distribution of the five ion species ${ }^{238} \mathrm{U}^{q+},{ }^{197} \mathrm{Au}^{q+},{ }^{181} \mathrm{Ta}^{q+},{ }^{132} \mathrm{Xe}^{q+}$, and ${ }^{84} \mathrm{Kr}^{q+}$ after the stripping was calculated. The initial charge state was chosen to be the minimum value allowed by GLOBAL, which means $q_{\max }-28$, or, if possible, their charge state assumed for operation in SIS100 (see Sec. VA). Various materials of different target thickness were considered in order to get optimal parameters of the stripping foil. The aim was to obtain the maximum possible stripping for all projectiles. Medium $Z$ materials (from $\mathrm{Al}$ to $\mathrm{Cu}$ ) are optimal for the efficient stripping of wide range of projectiles at different energies [56]. This was also

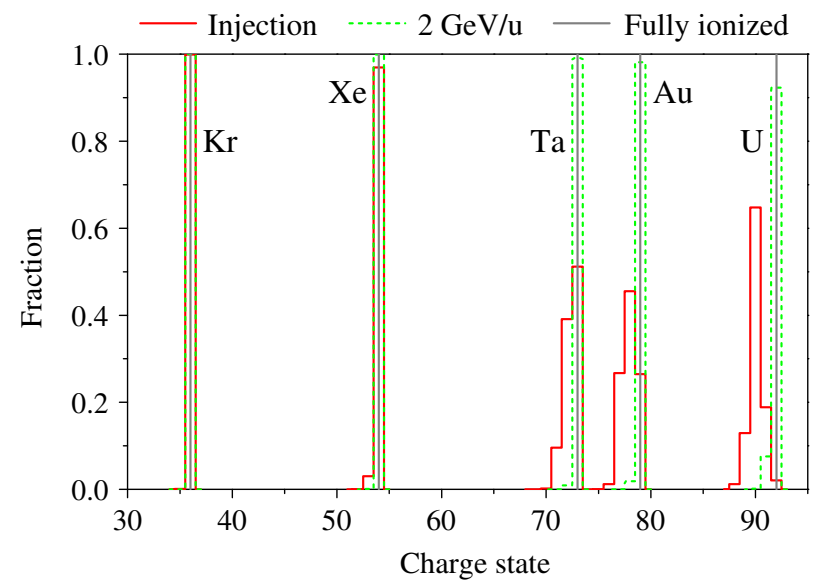

FIG. 12. Equilibrium charge state distribution of the ${ }^{238} \mathrm{U}^{q+}$, ${ }^{197} \mathrm{Au}^{q+},{ }^{181} \mathrm{Ta}^{q+},{ }^{132} \mathrm{Xe}^{q+}$, and ${ }^{84} \mathrm{Kr}^{q+}$ partially stripped ions interacting with $500 \mu \mathrm{m}$ thick titanium foil at injection energy and at $2 \mathrm{GeV} / \mathrm{u}$ with the marked fully ionized state of the ions.

confirmed by our calculations using GLOBAL, and $500 \mu \mathrm{m}$ thick titanium foil was found to be optimal for collimation of partially stripped ions in SIS100. All considered primary ion species reach in the foil of given thickness the equilibrium charge state distribution. The equilibrium charge state distribution of the ions was calculated at the beam injection energy and at $2 \mathrm{GeV} / \mathrm{u}$, which is the maximum energy allowed by GLOBAL. Results are presented in Fig. 12.

In the case of the lighter ions (from ${ }^{132} \mathrm{Xe}^{q+}$ and ${ }^{84} \mathrm{Kr}^{q+}$ ), it can be seen that there is practically no dependence on energy and almost all ions become fully ionized after interaction with the foil. For the heavier ions $\left({ }^{181} \mathrm{Ta}^{q+}\right.$, ${ }^{197} \mathrm{Au}^{q+}$, and ${ }^{238} \mathrm{U}^{q+}$ ), a noticeable charge state distribution was obtained at injection energies. The lowest charge state was observed in the case of ${ }^{238} \mathrm{U}$ ions and is equal to $q_{\max }-5$. At a higher energy of $2 \mathrm{GeV} / \mathrm{u}$, nearly all primary ions become fully ionized. Minor charge state distribution can be seen in the case of ${ }^{238} \mathrm{U}$, where less than $10 \%$ of the ions reach $q_{\max }-1$. But practically all projectiles can be considered for all energies as fully ionized after interaction with the stripping foil in comparison with their initial charge state.

\section{Particle tracking of stripped ions}

Particle tracking of the stripped ions deflection is rather simple and quick, since they pass only a few optical elements. Simulations were done again by using MAD-X. At least two stripping foils are needed for the primary stage of the collimation system: one in the horizontal and one in the vertical plane. The transverse position of the stripping foils is about $3.5 \sigma$ of the ${ }^{238} \mathrm{U}^{28+}$ beam distribution at injection energy and is larger for other ion species. The scattering of the ions by the stripping foil and the rms values of their angles, $\theta_{\text {rms }}$, were calculated by using 
FLUKA. In the case of the ${ }^{238} \mathrm{U}^{92+}$ ions, the $\theta_{\text {rms }}$ values vary between 1.96 and $0.22 \mathrm{mrad}$ for injection and extraction energy, respectively. The $\theta_{\text {rms }}$ values of the ${ }^{84} \mathrm{Kr}^{36+}$ ions vary between 0.92 and $0.14 \mathrm{mrad}$ for injection and extraction energy, respectively.

The first warm quadrupole of the doublet downstream of the stripping foil in the slow extraction area of SIS100 is defocusing in the horizontal plane. Because of that, the horizontal secondary collimator has to be located in the middle of the doublet. In the vertical plane, particles are first focused by the first and then defocused by the second quadrupole, respectively. For this reason, the vertical secondary collimator has to be placed behind the doublet.

The deflection of ${ }^{238} \mathrm{U}^{92+}$ and ${ }^{84} \mathrm{Kr}^{36+}$ ions with the initial charge states ${ }^{238} \mathrm{U}^{28+}$ and ${ }^{84} \mathrm{Kr}^{17+}$ stripped by the foil is presented in Fig. 13. The tracks of other stripped primary ion species are between those ones of ${ }^{238} \mathrm{U}^{92+}$ and ${ }^{84} \mathrm{Kr}^{36+}$ according to different charge exchange ratio $q_{e}$. The charge exchange ratio is defined as $q_{e}=\left(q_{s} / q_{i}\right)$, where $q_{i}$ is the initial charge state of the primary ions and $q_{s}$ is the charge state of the ions stripped by the foil. Only particle tracking of the ions which are fully ionized after the interaction with the stripping foil can be seen in Fig. 13. However, tracking of other charge states after the stripping presented in Fig. 12 showed practically no difference. It is caused by the fact that the charge state distribution of all considered ions is close to the fully ionized state. The scattering by the stripping foil was calculated at the injection energies where $\theta_{\text {rms }}$ values are the largest.
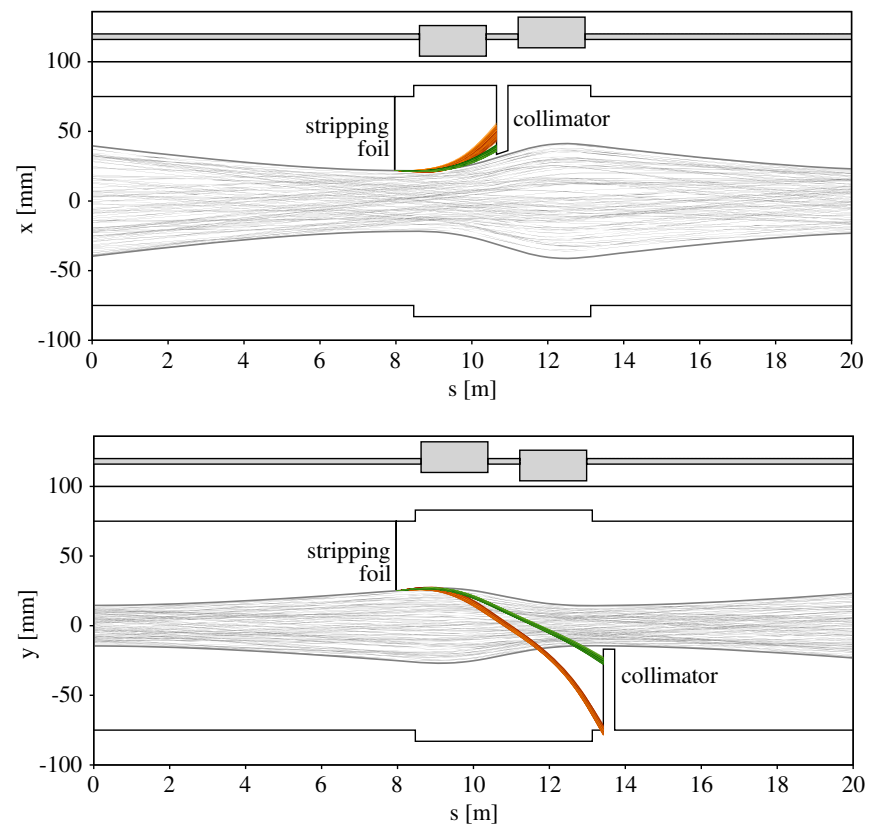

FIG. 13. Tracks of the ${ }^{238} \mathrm{U}^{92+}$ (orange) and ${ }^{84} \mathrm{Kr}^{36+}$ (green) ions deflected in the horizontal (above) and vertical (below) plane by the quadrupole field. The initial primary ions of charge states ${ }^{238} \mathrm{U}^{28+}$ and ${ }^{84} \mathrm{Kr}^{17+}$ were stripped by using the $500 \mu \mathrm{m}$ thick titanium foil.
TABLE IV. Dependence of the charge state distribution on the impact parameter. Fully ionized states are ${ }^{84} \mathrm{Kr}^{36+},{ }^{132} \mathrm{Xe}^{54+}$, and ${ }^{238} \mathrm{U}^{92+}$.

\begin{tabular}{lrcr}
\hline \hline & \multicolumn{3}{c}{ Charge states $q$} \\
\cline { 2 - 4 } Impact parameter $[\mu \mathrm{m}]$ & ${ }^{84} \mathrm{Kr}^{q+}$ & ${ }^{132} \mathrm{Xe}^{q+}$ & ${ }^{238} \mathrm{U}^{q+}$ \\
\hline 10 & 36 & $53-54$ & $88-92$ \\
1 & 36 & $53-54$ & $88-92$ \\
0.1 & $35-36$ & $52-54$ & $87-92$ \\
0.01 & $30-36$ & $44-53$ & $75-87$ \\
\hline \hline
\end{tabular}

A fraction of the partially stripped ions may not be able to pass through the full thickness of the stripping foil due to a small impact parameter. For the light ions, the equilibrium charge state distribution can be reached by using a much thinner stripping foil than the one chosen for the collimation in SIS100 [56]. The charge state distribution of heavy ions which pass through only a small part of the foil thickness can be shifted towards lower values than those presented in Fig. 12. However, the electrons which occupy outer shells of the heavy ions are stripped right after passing through a very thin material, and the desired charge state distribution can still be reached [56].

The charge state distribution of the ${ }^{84} \mathrm{Kr}^{q+},{ }^{132} \mathrm{Xe}^{q+}$, and ${ }^{238} \mathrm{U}^{q+}$ ions after passing through the stripping foil was calculated for the impact parameters from 10 down to $0.01 \mu \mathrm{m}$. The charge state distribution was calculated at injection energy. The results are presented in Table IV. For the three ions, there is a range of the charge states which occur with the probability higher than $10^{-3}$ after the stripping at a given impact parameter. A noticeable difference from the results shown in Fig. 12 is only at the smallest impact parameter. Nevertheless, the stripped ions are in this case also sufficiently deflected by the magnetic field and then collimated.

\section{SUMMARY AND CONCLUSIONS}

Various aspects of the beam halo collimation in heavy ion synchrotrons were studied, using the projected SIS100 as an example case. The interaction processes of the fully stripped ions with the primary collimator, such as scattering, momentum loss, and inelastic nuclear interaction, were investigated by using the simulation code FLUKA. The simulations were performed for various ion species from ${ }^{1} \mathrm{H}^{+}$up to ${ }^{238} \mathrm{U}^{92+}$. As a reference quantity for the calculations, the magnetic rigidity was chosen. The secondary collimators are for this study considered to be ideal absorbers.

It was found that at low rigidities the scattering angles for the heavier ions are larger than for protons. Towards higher rigidities, the scattering angles for protons and heavier ions start to coincide, and above $20 \mathrm{Tm}$ the values are all very similar. 
On the contrary to the scattering, the momentum losses in the primary collimator depend drastically on the ion species. This is not an issue for the single-pass collimation efficiency. However, the momentum losses are the main source of difference between the multipass collimation efficiency of light and heavy ions. Because of the considerably higher momentum losses, multiple transitions through the collimation system are not possible for the heaviest ions. It is because they are lost in highly dispersive arc sections of the accelerator downstream the collimation system.

Inelastic nuclear interactions include, in particular, hadronic fragmentation and EMD. The probability of the inelastic nuclear interaction in the primary collimator is of the order of a few percent for light ions. The probability increases with increasing atomic number, mass number, and energy of the primary ion. The EMD is important especially for high energy heavy ions. Deflection angles of the secondary particles are larger than for the primary particles. It is therefore assumed that their single-pass efficiency is also higher. A detailed calculation of the secondary particles production and their trajectories is a subject of the follow-up study.

The single-pass and multipass collimation efficiency for light and heavy ion beams in SIS100 was determined by using particle-tracking simulations performed by MAD-X. It was found that the single-pass efficiency is between $60 \%$ and $70 \%$ for all considered ion species at injection energy. The multipass efficiency at injection energy is higher than $99 \%$ from protons up to ${ }^{20} \mathrm{Ne}^{10+}$. The efficiency starts to decrease for ${ }^{40} \mathrm{Ar}^{18+}$ and drops to the level of the singlepass efficiency for ${ }^{132} \mathrm{Xe}^{54+}$. This is due to the substantially higher momentum losses of heavy ions in the primary collimator in comparison to protons and light ions. Because of this fact, multiple transitions through the collimation system do not exist, and the heavy ions which were not collimated are then lost in the arc sections.

The multipass collimation efficiency of heavy ions in SIS100 is significantly improved with the help of the cryocatchers. They are part of the special collimationpumping system originally designed for the interception of partially stripped ions after interaction with residual gas molecules. Because of the cryocatchers, the $99 \%$ efficiency level is reached up to ${ }^{132} \mathrm{Xe}^{54+}$, and even for ${ }^{238} \mathrm{U}^{92+}$ the efficiency remains at almost $90 \%$ at injection energy. In consequence of using cryocatchers in SIS100, the multipass efficiency is also not significantly affected by the collimation system adjustment, lattice imperfections, and beam parameters.

The collimation of partially stripped ions relies on the charge state change using a stripping foil. The stripped ions are then deflected by two warm quadrupoles in the slow extraction area of SIS100. For this reason, the performance of the collimation system is almost independent of the machine parameters or errors.
The equilibrium charge state in a thin titanium foil was obtained from GLOBAL for selected partially stripped ions from ${ }^{84} \mathrm{Kr}^{17+}$ up to ${ }^{238} \mathrm{U}^{28+}$ at injection energy and at $2 \mathrm{GeV} / \mathrm{u}$. The results indicate that all projectiles are close to the fully ionized state after the stripping. The tracking of the stripped ions downstream of the foil was performed again with mad-x. The simulations showed that all the considered partially stripped ions can be deflected effectively in the quadrupole field towards the secondary collimators.

\section{ACKNOWLEDGMENTS}

This work was supported by the German federal ministry of education and research (BMBF) under Contract No. 05P12RDRBM.

[1] K. Wittenburg, in Proceedings of the CAS-CERN Accelerator School: Course on Beam Diagnostics, Dourdan, France, 2008, edited by D. Brandt (CERN, Geneva, 2009), p. 557.

[2] H. D. Zhang, R. B. Fiorito, A. G. Shkvarunets, R. A. Kishek, and C. P. Welsch, Phys. Rev. ST Accel. Beams 15, 072803 (2012).

[3] A. V. Fedotov, Nucl. Instrum. Methods Phys. Res., Sect. A 557, 216 (2006).

[4] K. G. Sonnad and J. R. Cary, Phys. Rev. ST Accel. Beams 8, 064202 (2005).

[5] I. Strašík, E. Mustafin, and M. Pavlovič, Phys. Rev. ST Accel. Beams 13, 071004 (2010).

[6] A. Priebe et al., in Proceedings of the 2nd International Particle Accelerator Conference IPAC'11, San Sebastian, Spain, 2011, edited by C. Petit-Jean-Genaz (EPS-AG, Spain, 2011), p. 2388.

[7] E. Mahner, Phys. Rev. ST Accel. Beams 11, 104801 (2008).

[8] E. Mustafin, T. Seidl, A. Plotnikov, I. Strašík, M. Pavlovič, M. Miglierini, S. Stanček, A. Fertman, and A. Lančok, Radiat. Eff. Defects Solids 164, 460 (2009).

[9] P. Spiller et al., in Proceedings of the 4th International Particle Accelerator Conference IPAC'13, Shanghai, China, 2013, edited by C. Petit-Jean-Genaz (JACoW, Shanghai, 2013), p. 3782.

[10] O. Boine-Frankenheim, in Proceedings of the 1st International Particle Accelerator Conference IPAC'10, Kyoto, Japan, 2010, edited by C. Petit-Jean-Genaz (ICR, Kyoto, 2010), p. 2430.

[11] J. B. Jeanneret, Phys. Rev. ST Accel. Beams 1, 081001 (1998).

[12] T. Trenkler and J. B. Jeanneret, Part. Accel. 50, 287 (1995).

[13] R. Aßmann et al. (LHC Collimation Team), in Proceedings of the 46th ICFA Advanced Beam Dynamics Workshop on High-Intensity and High-Brightness Hadron Beams HB2010, Morschach, Switzerland, 2010, edited by A. Adelmann, J. Chrin, M. Marx, V. R. W. Schaa, and M. Seidel (PSI, Villigen, 2011), p. 21. 
[14] R. Bruce et al., Phys. Rev. ST Accel. Beams 17, 081004 (2014).

[15] B. Salvachua et al., in Proceedings of the 2012 Evian Workshop on LHC Beam Operation, Evian, France, 2012, edited by B. Goddard and S. Dubourg (CERN, Geneva, 2012), p. 155.

[16] K. Yamamoto, Phys. Rev. ST Accel. Beams 11, 123501 (2008).

[17] T. Koseki, Prog. Theor. Exp. Phys. (2012) $02 B 004$.

[18] B. C. Brown, P. Adamson, D. Capista, W. Chou, I. Kourbanis, D. K. Morris, K. Seiya, G. H. Wu, and M.-J. Yang, Phys. Rev. ST Accel. Beams 16, 071001 (2013).

[19] N. Mokhov et al., J. Instrum. 6, T08005 (2011).

[20] N. Catalan-Lasheras, Y. Y. Lee, H. Ludewig, N. Simos, and J. Wei, Phys. Rev. ST Accel. Beams 4, 010101 (2001).

[21] M. Seidel, Deutsches Elektronen-Synchrotron (DESY) Report No. DESY 94-103, 1994 (http://ccdb5fs.kek.jp/ cgi-bin/img/allpdf?199409031).

[22] K.-H. Mess and M. Seidel, Nucl. Instrum. Methods Phys. Res., Sect. A 351, 279 (1994).

[23] T. Wei and Q. Qin, Nucl. Instrum. Methods Phys. Res., Sect. A 566, 212 (2006).

[24] J. Y. Tang, J. F. Chen, and Y. Zou, Phys. Rev. ST Accel. Beams 14, 050103 (2011).

[25] R. Bruce, R. W. Aßmann, G. Bellodi, C. Bracco, H. H. Braun, S. Gilardoni, E. B. Holzer, J. M. Jowett, S. Redaelli, and T. Weiler, Phys. Rev. ST Accel. Beams 12, 011001 (2009).

[26] G. Bellodi, R. Aßmann, R. Bruce, M. Cauchi, J. M. Jowett, G. Valentino, and D. Wollmann, in Proceedings of the 2 nd International Particle Accelerator Conference IPAC'11, San Sebastian, Spain, 2011, edited by C. Petit-Jean-Genaz (EPS-AG, San Sebastian, 2011), p. 1813.

[27] V. M. Biryukov, Y. A. Chesnokov, and V. I. Kotov, Crystal Channeling and Its Applications at High Energy Accelerators (Springer-Verlag, Berlin, 1997).

[28] A. G. Afonin et al., Phys. Rev. Lett. 87, 094802 (2001).

[29] R. P. Fliller III, A. Drees, D. Gassner, L. Hammons, G. McIntyre, S. Peggs, D. Trbojevic, V. Biryukov, Y. Chesnokov, and V. Terekhov, Phys. Rev. ST Accel. Beams 9, 013501 (2006).

[30] W. Scandale et al., Phys. Rev. Lett. 102, 084801 (2009).

[31] W. Scandale et al., Phys. Lett. B 703, 547 (2011).

[32] G. Stancari, A. Valishev, G. Annala, G. Kuznetsov, V. Shiltsev, D. A. Still, and L. G. Vorobiev, Phys. Rev. Lett. 107, 084802 (2011).

[33] V. Shiltsev et al., Phys. Rev. ST Accel. Beams 11, 103501 (2008).

[34] J. Y. Tang, G. H. Wei, and C. Zhang, Nucl. Instrum. Methods Phys. Res., Sect. A 572, 601 (2007).

[35] A. I. Drozhdin, M. A. Kostin, and N. V. Mokhov, in Proceedings of the 2005 Particle Accelerator Conference PAC05, Knoxville, Tennessee, USA, 2005, edited by C. Horak (IEEE, New York, 2005), p. 2372.

[36] L. Bozyk, P. Spiller, and H. Kollmus, in Proceedings of the 3rd International Particle Accelerator Conference IPAC'12, New Orleans, Louisiana, USA, 2012, edited by C. Petit-Jean-Genaz (IEEE, Piscataway, NJ, 2012), p. 3237.

[37] P. Li et al., Phys. Rev. ST Accel. Beams 17, 084201 (2014).

[38] S. Di Mitri, Phys. Rev. ST Accel. Beams 13, 052801 (2010).

[39] T. T. Böhlen, F. Cerutti, M. P. W. Chin, A. Fassò, A. Ferrari, P. G. Ortega, A. Mairani, P. R. Sala, G. Smirnov, and V. Vlachoudis, Nucl. Data Sheets 120, 211 (2014).

[40] A. Fasso, A. Ferrari, J. Ranft, and P. R. Sala, Reports No. CERN-2005-10, No. INFN/TC_05/11, and No. SLAC-R-773, 2005.

[41] 14th FLUKA Course, Dresden, Germany, 2013 (http://www .fluka.org).

[42] L. Deniau et al. (MAD development team), MADMethodical Accelerator Design (http://mad.web.cern.ch/ mad).

[43] W. Herr and F. Schmidt, CERN Report No. CERN-AB2004-027-ABP, 2004 (http://madx.web.cern.ch/madx/doc/ madx_primer.pdf).

[44] J. Beringer et al. (Particle Data Group), Phys. Rev. D 86, 010001 (2012).

[45] V. Andersen et al., Adv. Space Res. 34, 1302 (2004).

[46] R. K. Tripathi, F. A. Cucinotta, and J. W. Wilson, Nucl. Instrum. Methods Phys. Res., Sect. B 117, 347 (1996).

[47] J. W. Norbury, Phys. Rev. C 47, 406 (1993).

[48] J. W. Norbury and A. Adamczyk, Nucl. Instrum. Methods Phys. Res., Sect. B 254, 177 (2007).

[49] H. H. Braun, A. Fassò, A. Ferrari, J. M. Jowett, P. R. Sala, and G. I. Smirnov, Phys. Rev. ST Accel. Beams 17, 021006 (2014).

[50] National Physical Laboratory, Tables of Physical, and Chemical Constants, Kaye, and Laby Online Version 1.1, 2008 (http://www.kayelaby.npl.co.uk).

[51] G. Valentino, R. Aßmann, R. Bruce, F. Burkart, V. Previtali, S. Redaelli, B. Salvachua, G. Stancari, and A. Valishev, Phys. Rev. ST Accel. Beams 16, 021003 (2013).

[52] R. Aßmann, in Handbook of Accelerator Physics, and Engineering, 2nd ed., edited by A. W. Chao, K. H. Mess, M. Tigner, and F. Zimmermann (World Scientific, Singapore, 2013), Chap. 3.3.11.

[53] G. Franchetti, I. Hofmann, S. Sorge, and V. Kapin, in Proceedings of the 23rd Particle Accelerator Conference PAC09, Vancouver, Canada, 2009, edited by M. Comyn (IEEE, Piscataway, NJ, 2009), p. 3242.

[54] H.H. Gutbrod, FAIR-Baseline Technical Report Vol. 2, 2006 (http://www.fair-center.eu/fileadmin/fair/ publications_FAIR/FAIR_BTR_2.pdf).

[55] A. Smolyakov, E. Mustafin, N. Pyka, and P. Spiller, in Proceedings of the 11th European Particle Accelerator Conference EPAC'08, Genoa, Italy, 2008, edited by C. Petit-Jean-Genaz (EPS-AG, Genoa, Italy, 2008), p. 3602.

[56] C. Scheidenberger, Th. Stohlker, W. E. Meyerhof, H. Geissel, P. H. Mokler, and B. Blank, Nucl. Instrum. Methods Phys. Res., Sect. B 142, 441 (1998).

[57] W. E. Meyerhof, GLOBAL (http://web-docs.gsi.de/ weick/ charge_states/). 